Journal of Student Wellbeing

December 2010, Vol. 4(2), 1-2.

\title{
Editorial: Sport, health and physical education special edition
}

\author{
Murray Drummond \\ Associate Professor \\ School of Education \\ Flinders University \\ South Australia
}

This special edition of the Journal of Student Wellbeing emerges out of the Sport, Health and Physical Education (SHAPE) conference held at Flinders University in 2010. The conference was organised and run by the SHAPE research group, which is located within the School of Education at Flinders University. It was designed to bring together national and international academics to share their research and knowledge around children's health and wellbeing within the context of sport, health and physical education settings. Approximately 150 delegates attended. The conference themes were divided into four key areas: (i) physical activity research, (ii) physical activity programs in schools, (iii) nutrition education and (iv) physical education.

The papers within this special edition are indicative of the presentations conducted by a number of the presenters at the conference. The first paper is by one of the invited keynote presenters, Associate Professor Jenny O’Dea from the University of Sydney. Jenny is an internationally renowned academic in the area of nutrition education, obesity and body image. She has provided a paper on 'Studies of obesity, body image and related health issues among Australian adolescents: how can programs in schools interact with and complement each other?' The paper details recent studies of body weight, obesity, body image and related health issues among Australian adolescents and the ways in which subsequent nutrition and physical activity programs in schools can interact with and complement each other, rather than contradicting or competing with each other.

The second paper by M Drummond, C Drummond, Dollman and Abery titled 'Physical activity from early childhood to adolescence: a literature review of issues and interventions in disadvantaged populations' investigates the major issues around physical activity confronting children and families in disadvantaged populations. It explores multi-faceted interventions within schools and in families. 
Shane Pill's paper on 'Sport literacy: it's not just about learning to play sport via "textbook techniques"' challenges traditional approaches to teaching sport within physical education. Indeed, as Shane clearly articulates, sport and physical education are often used as almost interchangeable terms; however, sport is not physical education. This paper identifies ways in which sport can be taught through a sport literacy model that can achieve educational outcomes.

Dr Claire Drummond's paper, 'Using nutrition education and cooking classes in primary schools to encourage healthy eating', provides empirical research data using the voices of children and teachers within focus group interviews prior to, and following, nutrition education workshops in schools. The research provides evidence that nutrition workshops in schools can have many positive benefits for children.

The final paper by Velardo, Elliott, Filiault and M Drummond on 'The role of health literacy in parents' decision making in children's sporting participation' challenges the traditional ideology surround parents' motivation for engaging their children in sport through competition. They claim that taking a health literacy approach and basing participation on the development of healthy life skills will be a more sustainable approach to sport and physical activity participation throughout childhood and beyond.

This edition has drawn together a group of academics with varied backgrounds and research emphases, yet the one thing that gels them collectively is childhood and student wellbeing through physical activity, nutrition education and sporting engagement. I hope that these articles will challenge and engage readers ranging from academics to teachers and tertiary students to reflect upon the wide-ranging issues that impact on children and their health in contemporary society. 